\title{
Momentum Dependence of Orbital Excitations in Mott-Insulating Titanates
}

\author{
C. Ulrich, ${ }^{1}$ L. J. P. Ament, ${ }^{2}$ G. Ghiringhelli, ${ }^{3}$ L. Braicovich, ${ }^{4}$ M. Moretti Sala, ${ }^{4}$ N. Pezzotta, ${ }^{4}$ T. Schmitt, ${ }^{5}$ G. Khaliullin, ${ }^{1}$ \\ J. van den Brink, ${ }^{2,6}$ H. Roth, ${ }^{7}$ T. Lorenz, ${ }^{7}$ and B. Keimer ${ }^{1}$ \\ ${ }^{1}$ Max-Planck-Institut für Festkörperforschung, D-70569 Stuttgart, Germany \\ ${ }^{2}$ Institute-Lorentz for Theoretical Physics, Universiteit Leiden, 2300 RA Leiden, The Netherlands \\ ${ }^{3}$ CNR/INFM COHERENTIA and Dipartimento di Fisica, Politecnico di Milano, 20133 Milano, Italy \\ ${ }^{4}$ CNR/INFM SOFT and Dipartimento di Fisica, Politecnico di Milano, 20133 Milano, Italy \\ ${ }^{5}$ Swiss Light Source, Paul Scherrer Institut, CH-5232 Villigen PSI, Switzerland \\ ${ }^{6}$ Stanford Institute for Materials and Energy Sciences, SLAC, Menlo Park, California 94025, USA \\ ${ }^{7}$ II. Physikalisches Institut, Universität zu Köln, 50937 Köln, Germany
}

(Received 17 March 2009; published 4 September 2009)

\begin{abstract}
High-resolution resonant inelastic x-ray scattering has been used to determine the momentum dependence of orbital excitations in Mott-insulating $\mathrm{LaTiO}_{3}$ and $\mathrm{YTiO}_{3}$ over a wide range of the Brillouin zone. The data are compared to calculations in the framework of lattice-driven and superexchange-driven orbital ordering models. A superexchange model in which the experimentally observed modes are attributed to two-orbiton excitations yields the best description of the data.
\end{abstract}

DOI: 10.1103/PhysRevLett.103.107205

Valence electrons in transition metal oxides exhibit a large variety of ordering phenomena that are associated with unusual macroscopic properties [1]. In order to elucidate the origin of these properties, experimental research beginning in the 1950's has systematically investigated the relationship between the occupation of $d$ orbitals on metal ions and the magnetic ordering pattern. The leading paradigm for the interpretation of these data is the "Goodenough-Kanamori" framework, which is based on the assumptions that the orbital occupation is determined by electron-lattice interactions alone, and that the relative orientation of orbitals on neighboring sites controls the exchange interactions between magnetic ions [1]. While experiments on most transition metal oxides are in accordance with this paradigm, it has been called into question by recent data on the Mott insulators $\mathrm{LaTiO}_{3}$ and $\mathrm{YTiO}_{3}$ with the seemingly simple electron configuration $3 d^{1}$. Not only does the spin dynamics of these compounds appear to defy a quantitative description in terms of the standard lattice-driven orbital ordering scenario [2,3], thermodynamic data [4] on $\mathrm{LaTiO}_{3}$ have even been interpreted as evidence of a novel spin-orbital liquid state [5] that is qualitatively incompatible with this scheme. An alternative approach that emphasizes the many-body superexchange coupling between spin and orbital degrees of freedom while treating the local electron-lattice interaction as a perturbation, can explain some of these experimental findings [5,6]. Other experiments, however, have been interpreted in terms of the rigid orbital ordering pattern predicted by the Goodenough-Kanamori framework [713]. Further experimental work is therefore required in order to discriminate between the competing theoretical scenarios and to develop a comprehensive understanding of the electronic structure of these prototypical orbitally degenerate Mott insulators.
PACS numbers: 75.30.Et, 71.70.Ch, 75.50.Dd, 78.70.Ck

Momentum-resolved spectroscopies, which associate specific energy scales directly with the chemical bonding pattern, are among the most powerful experimental probes of transition metal oxides. In particular, the momentum $q$ dependence of low-energy $(\sim 10 \mathrm{meV})$ spin excitations determined by neutron spectroscopy [2,3] and high-energy $(\sim 1 \mathrm{eV})$ charge excitations determined by electron [14], photoemission [15], and x-ray [16] spectroscopies has yielded deep insights into the electronic structure of Mott insulators. Recently, orbital excitations with intermediate energies $(\sim 250 \mathrm{meV})$ have been detected by Raman [17] and infrared [18] spectroscopies in Mott-insulating titanates, but these methods are limited to $q=0$. Because of the much larger photon wave vectors involved in the scattering process, resonant inelastic $\mathrm{x}$-ray scattering (RIXS) can in principle serve as a probe of the momentum dependence of the orbital excitations [19-21]. This is particularly informative for the titanates, because of sharply divergent predictions by the two theoretical scenarios. Whereas orbital excitations in the lattice-driven theory are weakly dispersive because their energies are dominated by the local Jahn-Teller coupling [9-11], the superexchange model predicts collective modes with a substantial dispersion [6]. Instrumental constraints have, however, thus far precluded RIXS experiments on orbital excitations with $q \neq 0$.

We have overcome these limitations at the newly developed ADRESS beam line at the Swiss Light Source [22,23] and report RIXS data with photon energies near the Ti $L_{2,3}$ absorption edge of $\mathrm{LaTiO}_{3}$ and $\mathrm{YTiO}_{3}$ over a large fraction of the Brillouin zone. The experiments were performed on high-quality $\mathrm{YTiO}_{3}$ and $\mathrm{LaTiO}_{3}$ single crystals (grown as described in Ref. [7]) with magnetic transition temperatures $T_{C}=27 \mathrm{~K}$ and $T_{N}=146 \mathrm{~K}$, respectively. The $\mathrm{YTiO}_{3}\left(\mathrm{LaTiO}_{3}\right)$ crystal was untwinned (partly twinned 
in the $a b$ plane of the orthorhombic space group Pbnm). Since the orthorhombic distortion is small, we use the pseudocubic notation, i.e., $a^{\prime}=a / \sqrt{2}, b^{\prime}=b / \sqrt{2}$, and $c^{\prime}=c / 2$. The crystals were cut and polished with surface normals along the [110] and [100] directions, respectively, for $\mathrm{LaTiO}_{3}$ and $\mathrm{YTiO}_{3}$. This allowed investigations of orbital excitations with momenta along these two directions in the Brillouin zone. The RIXS data were taken at the ADRESS beam line, where an energy resolution of $55 \pm 2 \mathrm{meV}$ was obtained using a combination of focusing elements and high-precision optical gratings [22,23]. $\mathrm{X}$-ray absorption spectra taken to select the energy for the RIXS experiment were identical to those presented in Ref. [20]. To study the momentum dependence of the orbital excitations, the incident photon energy was adjusted to either 452.0 or $454.6 \mathrm{eV}$ at the Ti $L_{3}$ edge (corresponding to excitations from the $2 p_{3 / 2}$ core level into the $3 d t_{2 g}$ and $e_{g}$ levels, respectively), and the scattering angle $2 \theta$ was changed in a range between $50^{\circ}$ and $130^{\circ}$. Data were taken at $T=13 \mathrm{~K}$ (i.e., in the magnetically ordered states) and at
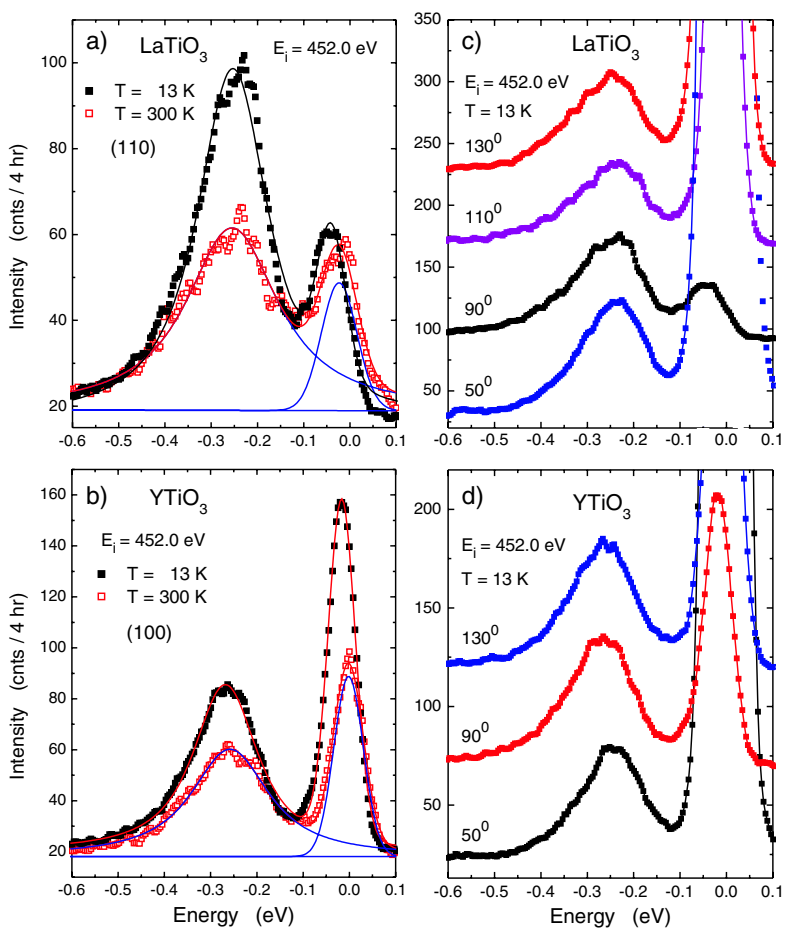

FIG. 1 (color online). RIXS spectra of (a) $\mathrm{LaTiO}_{3}$ and (b) $\mathrm{YTiO}_{3}$ at room temperature and at $T=13 \mathrm{~K}$. The spectra were taken at an incident energy $E_{i}=452.0 \mathrm{eV}$ (i.e., at the $t_{2 g}$ level of the $L_{3}$ excitation of the Ti ions) in horizontal polarization of the incident light at a scattering angle $2 \theta=90^{\circ}$. The lines are the results of fits to the Voigt line shape. (c), (d) Evolution of the RIXS spectra as a function of $2 \theta$. Note that the excitation momentum $q=2 k_{i} \sin \theta$, where $k_{i}$ is the incident photon wave vector. The data along [100] ([110]) thus span a range of 11.5 to $25 \%$ (8.5 to $17.5 \%$ ) of the diameter of the Brillouin zone. The weak features in the spectra arise from statistical fluctuations of the count rate, which were smoothed out by the detector readout algorithm. room temperature. The incident $\mathrm{x}$-ray beam was polarized either horizontally or vertically (parallel or perpendicular to the scattering plane, respectively). While excitations of comparable intensity were detected in both polarization geometries, elastic scattering arising from static disorder, surface effects, and partly from thermal diffuse scattering is strongly enhanced for vertical polarization. The elastic peak in vertical polarization was therefore used for calibrations of the photon energy (performed periodically between inelastic scans) and a determination of the energy resolution. The spectra shown below were taken in horizontal polarization. They are sums of partial spectra, each accumulated for $10 \mathrm{~min}$ for a total of $4 \mathrm{~h}$.

Figure 1 shows the $t_{2 g}$ excitation spectra (incident photon energy $452.0 \mathrm{eV}$ ) of $\mathrm{LaTiO}_{3}$ and $\mathrm{YTiO}_{3}$ at room temperature and at $T=13 \mathrm{~K}$ in horizontal polarization with $2 \theta=90^{\circ}$ [(panels (a),(b)]. In this scattering geometry the diffuse elastic peak is strongly suppressed, and the signal is dominated by inelastic scattering. All inelastic peaks appear on the energy-loss side, as expected because the excitation energy of $\sim 250 \mathrm{meV}$ greatly exceeds $k_{B} T$. The peaks disappear when the energy of the incident beam is tuned away from the resonance condition, demonstrating that they arise from excitations of the $3 d$ electrons of the Ti ions. The inelastic peaks sharpen with decreasing temperature, and their intensities increase. The same effect has also been observed by Raman light scattering [17]. Interestingly, the "elastic" peaks at $T=13 \mathrm{~K}$ are not centered at zero energy, but at $40(16) \mathrm{meV}$ for $\mathrm{LaTiO}_{3}\left(\mathrm{YTiO}_{3}\right)$. The shift closely corresponds to the energy range of (anti-) ferromagnetic magnons previously detected by neutron scattering [2,3]. At room temperature, where both compounds are paramagnetic, the peak centers shift to 22 (0) $\mathrm{meV}$, supporting an interpretation in terms of magnons. With further improvement of the energy resolution, RIXS may thus become widely applicable as a probe of dispersive magnon excitations.

The momentum dependence of the RIXS spectra at $T=$ $13 \mathrm{~K}$ is shown in Figs. 1(c) and 1(d). Kinematic and instrumental constraints restrict the $q$ range to $18.2 \%$ $(25.8 \%)$ of the Brillouin zone for $\mathrm{YTiO}_{3}$ along [100] $\left(\mathrm{LaTiO}_{3}\right.$ along [110]). Note that the Brillouin zone boundary is at $50 \%$. In a first step of a quantitative analysis, the data were fitted to Voigt profiles, which correspond to a convolution of a Lorentzian with the Gaussian spectrometer resolution [lines in Figs. 1(a) and 1(b)]. The resulting peak positions and amplitudes of both $t_{2 g}$ and $e_{g}$ excitations are plotted in Fig. 2, along with previously reported [17] data for $q=0$. Only a small variation of the peak positions is observed in the $q$ range covered by our experiment [Fig. 2(a)]. This behavior (which is very similar in both compounds) is incompatible with the strongly dispersive single-orbiton excitations predicted by the superexchange model. Our calculations below demonstrate, however, that the RIXS intensity of two-orbiton excitations can exceed that of single-orbiton modes. The intensity 

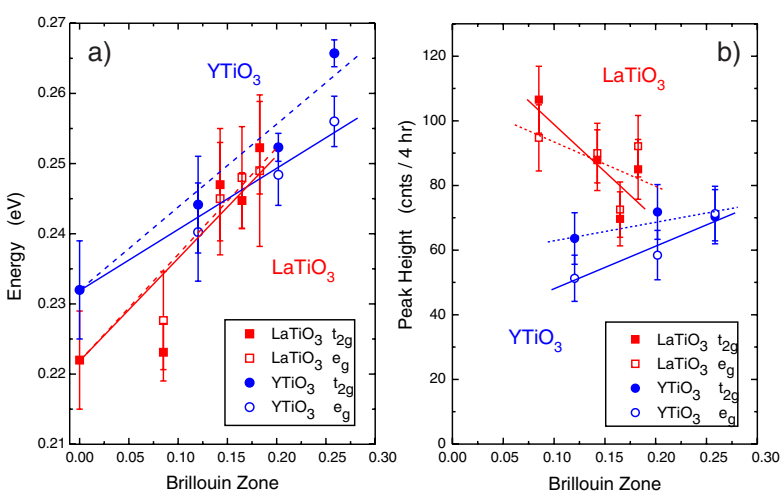

FIG. 2 (color online). Momentum $(q)$ dependence of (a) the energy and (b) the amplitude of the RIXS signal of $\mathrm{LaTiO}_{3}$ and $\mathrm{YTiO}_{3}$ at $T=13 \mathrm{~K} . q$ is given as a fraction of the size of the Brillouin zone (BZ) along the high-symmetry directions quoted in the text. Note that the BZ boundary is at $50 \%$. The $q=0$ data obtained from Raman light scattering [17] are also shown in (a). The lines are guides to the eye.

maximum of the two-orbiton continuum is determined by a convolution of two single-orbiton states with momenta adding to $q$, and its dispersion is expected to average out.

In order to obtain a quantitative description of the contribution of one- and two-orbiton excitations, we now consider the scattering processes in detail. We first focus on a superexchange model for $\mathrm{YTiO}_{3}$ (Ref. [6]), and then compare the results to those of a lattice-driven scenario. In RIXS, a $2 p$ electron of the Ti ion is lifted to the $3 d$ level, modifying the electronic environment. In particular, the core-hole potential effectively reduces the Coulomb repulsion $U$ by the amount $U_{c}$, and therefore modulates the superexchange interaction $J_{\mathrm{SE}}=4 t^{2} / U$. This exchangebond-modulation mechanism was successfully used to explain the observation [24] of two-magnon scattering by RIXS [25-27]. In the titanates, modification of $J_{\mathrm{SE}}$ causes either one or two orbital flips on neighboring Ti sites. Along the lines of Ref. [21], the following effective scattering operator can be derived:

$$
\hat{O}_{\mathbf{q}}=\sum_{i, \delta} e^{i \mathbf{q} \cdot \mathbf{R}_{i}}\left(J_{1} \hat{n}_{i+\delta}^{(\gamma)}+J_{2} \hat{A}_{i, i+\delta}^{(\gamma)}\right),
$$

where $J_{1}=\frac{t^{2}}{U-U_{c}}-\frac{t^{2}}{U}$ and $J_{2}=\frac{t^{2}}{U+U_{c}}+\frac{t^{2}}{U-U_{c}}-\frac{2 t^{2}}{U}$. The operators $\hat{n}_{i}^{(\gamma)}$ and $\hat{A}_{i, i+\delta}^{(\gamma)}$ were taken from Ref. [6] and contain the density and pseudospin one-half orbital operators whose explicit form depends on the spatial direction $\gamma=a, b, c$ of the exchange bonds. The first term $J_{1}$ represents single-site processes, whereas the second term acts on two sites. In terms of orbitons, the latter term only creates two orbitons, while the former can create both one and two orbitons. The single-orbiton contribution scales with the orbital order parameter which is strongly reduced [6] due to the orbital frustration inherent in the superexchange model. Therefore, the two-orbiton response dominates the scattering intensity. Moreover, for the [100] direction the $J_{1}$-scattering channel is forbidden for symmetry reasons, because the number of $y z$ orbitals is conserved on each [100] plane independently.

The results computed for the bond-modulation RIXS process are shown in Fig. 3(a). The scattering intensity was calculated using the orbital dispersion relation from Ref. [6], with the orbital exchange strength $r_{1} J_{\mathrm{SE}}=$ $75 \mathrm{meV}$ (close to the value of $60 \mathrm{meV}$ estimated in Ref. [6]). In order to facilitate comparison with the experimental data, we introduce an orbital damping $\gamma=30 \mathrm{meV}$ due to orbiton-orbiton (superexchange model) or orbitalphonon interactions (Jahn-Teller model). The spectra are further convoluted with the resolution function of the spectrometer. The line shapes of the calculated and measured RIXS spectra agree very well.

Figure 3(a) shows that the bond-modulation mechanism alone fails to describe the evolution of the absolute intensity of the RIXS signal with increasing $q$. However, there is another coupling mechanism that also creates orbital excitations. Namely, if the potential of the core-hole is not of $A_{1 g}$ symmetry, it locally shakes up the occupied orbitals, which can leave the $\mathrm{Ti}$ ions behind in an orbitally excited state. The corresponding scattering operator can be written in a general form as $\hat{O}_{\mathbf{q}}=\sum_{i} \hat{O}_{i} e^{i \mathbf{q} \cdot \mathbf{R}_{i}}$. Because of the octahedral symmetry of the $\mathrm{TiO}_{6}$ unit, the local scattering operator $\hat{O}_{i}$ can be decomposed into components $\left(\hat{f}_{\kappa}^{(j)}\right)_{i}$ transforming according to the rows $\kappa$ of the irreducible representations $j$ of the octahedral group:

$$
\hat{O}_{\mathbf{q}}=\sum_{j, \kappa} P_{j, \kappa} M_{j} \sum_{i}\left(\hat{f}_{\kappa}^{(j)}\right)_{i} e^{i \mathbf{q} \cdot \mathbf{R}_{i}} .
$$

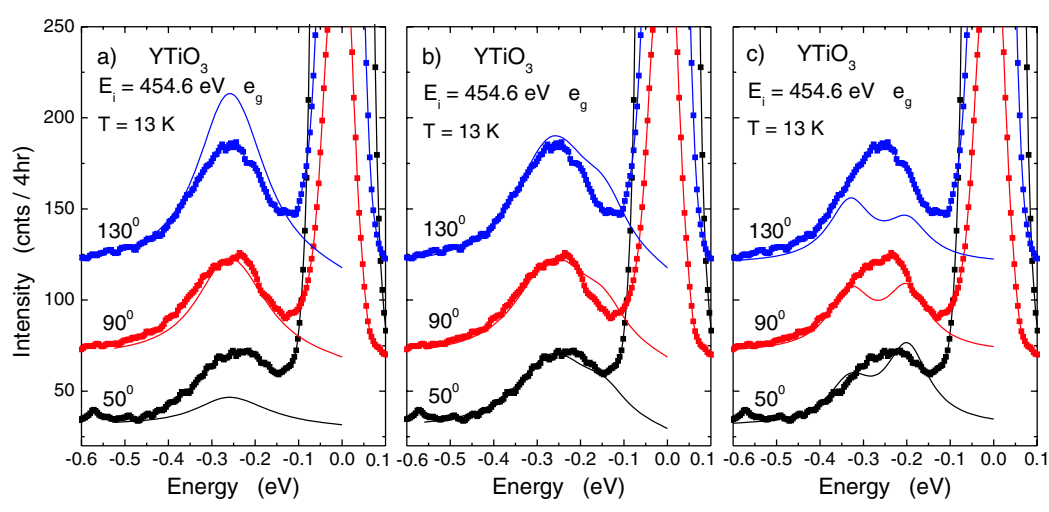

$107205-3$
FIG. 3 (color online). Comparison of the model calculations with the experimental RIXS data of $\mathrm{YTiO}_{3}$ for different scattering vectors $q$ along the [100] direction: (a) superexchange bond modulation, (b) local shakeup of collective orbital excitations, and (c) local crystalfield excitation model. 
The operators $\left(\hat{f}_{\kappa}^{(j)}\right)_{i}$ induce an orbital transition of certain symmetry, the polarization factors $P_{j, \kappa}$ depend on the experimental geometry. The factors $M_{j}$ depend on the multiplet structure of the intermediate states and can be obtained from cluster calculations. We take only up to quadrupole components of $\left(\hat{f}_{\kappa}^{(j)}\right)_{i}$ into account (the higher multipoles contribute much less), and assume that all $M_{j}$ are equal. The spectra of the operators $\left(\hat{f}_{\kappa}^{(j)}\right)_{\mathbf{q}}$ are calculated within the superexchange model. Figure 3(b) compares the result of the calculations with the experimental data. Here the energy scale $r_{1} J_{\mathrm{SE}}$ was adjusted to $80 \mathrm{meV}$. Both the lineshape and the scattering intensity of the observed inelastic peak are reproduced for all scattering vectors. The only noticeable difference is the weak shoulder that appears in the calculations at about $120 \mathrm{meV}$. This arises from single-orbiton excitations, which in this case are not forbidden for the [100] direction.

In addition to the orbital superexchange model, we calculated the RIXS intensity in the opposite scenario of strong orbital-lattice coupling. The orbital excitations are now local crystal-field transitions activated by the shakeup mechanism. We use the orbital ordering pattern and crystal-field splittings (200 and $330 \mathrm{meV}$ ) of Ref. [11] to obtain the spectra shown in Fig. 3(c). (Closely similar values were also obtained by other calculations $[8,18]$.) The crystal-field transitions are localized and do not show any dispersion, but due to the $q$ dependence of the transition matrix element, the intensity ratio of the peaks can vary. With increasing $q$, a shift of the overall spectral weight to higher energies is thus seen, as in the experimental data. However, the calculated spectrum is comprised of two well-separated peaks and is hence in poor agreement with the RIXS data, which show a single broad feature. An ad hoc reduction of the level splitting away from the predicted values $[8,11,18]$ could improve the agreement of the line shape, but this does not affect the $q$ dependence of the intensity, which exhibits a trend opposite to what is observed.

In conclusion, we have determined the momentum dependence of the RIXS spectra of $\mathrm{LaTiO}_{3}$ and $\mathrm{YTiO}_{3}$ and compared the results to quantitative predictions of the superexchange and crystal-field models. We found that the former model yields better agreement with the experimental data on $\mathrm{YTiO}_{3}$. On a qualitative level, we note that the $q$-dependent intensities of the orbiton excitations of $\mathrm{YTiO}_{3}$ and $\mathrm{LaTiO}_{3}$ [Fig. 2(b)] exhibit trends opposite to those of the corresponding spin excitations [2,3], as expected in superexchange models. Mixing of spin and orbital correlations by the superexchange interaction also accounts naturally for the strong temperature dependence of the RIXS intensity, which is difficult to explain in terms of localized crystal-field excitations. An open problem is the spectral weight of single-orbiton excitations, which is predicted to be larger than experimentally observed. A possible explanation is that we have assumed the spins to be fully saturated, while in $\mathrm{YTiO}_{3}$ the ordered moment is lower than $1 \mu_{B}$ even at zero temperature, presumably as a consequence of joint spin-orbital fluctuations. Such fluctuations are also expected to reduce the orbital order parameter, to which the single-orbiton weight is proportional.

We thank M. W. Haverkort for useful discussions. L. A. thanks the MPI-FKF, Stuttgart, for its hospitality. The crystal growth in Cologne was supported by the DFG through SFB608. This work was performed at the ADRESS beam line of the SLS (Paul Scherrer Institut) using the SAXES spectrometer developed jointly by Politecnico di Milano, SLS, and EPFL.

[1] For a review, see Y. Tokura and N. Nagaosa, Science 288, 462 (2000).

[2] B. Keimer et al., Phys. Rev. Lett. 85, 3946 (2000).

[3] C. Ulrich et al., Phys. Rev. Lett. 89, 167202 (2002).

[4] J.-G. Cheng, Y. Sui, J.-S. Zhou, J. B. Goodenough, and W. H. Su, Phys. Rev. Lett. 101, 087205 (2008).

[5] G. Khaliullin and S. Maekawa, Phys. Rev. Lett. 85, 3950 (2000).

[6] G. Khaliullin and S. Okamoto, Phys. Rev. Lett. 89, 167201 (2002); Phys. Rev. B 68, 205109 (2003).

[7] M. Cwik et al., Phys. Rev. B 68, 060401(R) (2003).

[8] R. Schmitz et al., Phys. Rev. B 71, 144412 (2005); Ann. Phys. (Leipzig) 14, 626 (2005).

[9] M.W. Haverkort et al., Phys. Rev. Lett. 94, 056401 (2005).

[10] M. Mochizuki and M. Imada, Phys. Rev. Lett. 91, 167203 (2003).

[11] E. Pavarini et al., Phys. Rev. Lett. 92, 176403 (2004); New J. Phys. 7, 188 (2005).

[12] I. V. Solovyev, Phys. Rev. B 74, 054412 (2006).

[13] T. Kiyama and M. Itoh, Phys. Rev. Lett. 91, 167202 (2003).

[14] J. Fink et al., J. Electron Spectrosc. Relat. Phenom. 117118, 287 (2001).

[15] A. Damascelli et al., Rev. Mod. Phys. 75, 473 (2003).

[16] See, e.g., Y. J. Kim et al., Phys. Rev. B 76, 155116 (2007).

[17] C. Ulrich et al., Phys. Rev. Lett. 97, 157401 (2006).

[18] R. Rückamp et al., New J. Phys. 7, 144 (2005).

[19] S. Ishihara and S. Maekawa, Phys. Rev. B 62, 2338 (2000); S. Ishihara, Phys. Rev. B 69, 075118 (2004).

[20] C. Ulrich et al., Phys. Rev. B 77, 113102 (2008).

[21] F. Forte, L. J. P. Ament, and J. van den Brink, Phys. Rev. Lett. 101, 106406 (2008).

[22] G. Ghiringhelli et al., Rev. Sci. Instrum. 77, 113108 (2006).

[23] For a description of the ADRESS beam line, see http:// sls.web.psi.ch/view.php/beamlines/adress/index.html

[24] J. P. Hill et al., Phys. Rev. Lett. 100, 097001 (2008); L. Braicovich et al., Phys. Rev. Lett. 102, 167401 (2009).

[25] J. van den Brink and M. van Veenendaal, Europhys. Lett. 73, 121 (2006); J. van den Brink, ibid. 80, 47003 (2007).

[26] L. J. P. Ament, F. Forte, and J. van den Brink, Phys. Rev. B 75, 115118 (2007); F. Forte, L. J. P. Ament, and J. van den Brink, ibid. 77, 134428 (2008).

[27] T. Nagao and J. I. Igarashi, Phys. Rev. B 75, 214414 (2007). 